\title{
Steroidogenic factor $1 /$ adrenal 4 binding protein transforms human bone marrow mesenchymal cells into steroidogenic cells
}

\author{
Tomoko Tanaka ${ }^{1}$, Shigeki Gondo', Taijiro Okabe ${ }^{1}$, Kenji Ohe ${ }^{1}$, Hisao Shirohzu', \\ Hidetaka Morinaga ${ }^{1}$, Masatoshi Nomura ${ }^{1}$, Kenzaburo Tani ${ }^{2}$, Ryoichi Takayanagi ${ }^{1}$, \\ Hajime Nawata ${ }^{3}$ and Toshihiko Yanase ${ }^{1}$ \\ ${ }^{1}$ Department of Medicine and Bioregulatory Science, Graduate School of Medical Science, ${ }^{2}$ Department of Molecular Genetics, Medical Institute of Bioregulation \\ and ${ }^{3}$ Graduate School of Medical Science, Kyushu University, Maidashi 3-1-1, Higashi-ku, Fukuoka 812-8582, Japan \\ (Correspondence should be addressed to T Yanase; Email: yanase @ intmed3.med.kyushu-u.ac.jp)
}

\begin{abstract}
Steroidogenic factor 1/adrenal 4 binding protein (SF-1/Ad4BP) is an essential nuclear receptor for steroidogenesis as well as for adrenal and gonadal gland development. Mesenchymal bone marrow cells (BMCs) contain pluripotent progenitor cells, which differentiate into multiple lineages. In a previous study, we reported that adenovirus-mediated forced expression of SF-1 could transform mouse primary long-term cultured BMCs into steroidogenic cells. For future clinical application, trials using human BMCs would be indispensable. In this study, we examined whether SF-1 could transform human BMCs into steroidogenic cells and compared the steroid profile of these cellswith that of mouse steroidogenic BMCs. Primary cultured human BMCs infected with adenovirus containing bovine SF-1 cDNA could produce progesterone, corticosterone, cortisol, dehydroepiandrosterone, testosterone, and estradiol. Such a mixed character of adrenal and gonadal steroid production in human BMCs was supported by the expressions of P450scc, 3 $\beta$ -

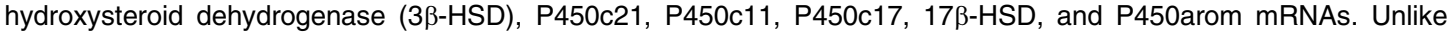
mouse steroidogenic BMCs, introduction of SF-1 into human BMCs caused dramatic inductions of both ACTH and LH receptors, thus leading to good responsiveness of the cells to ACTH and LH respectively. Importantly, among several factors that are known to be closely associated with adrenal and/or gonadal development, introduction of only SF-1 enabled the human BMCs to express P450scc and to produce cortisol and testosterone, suggesting that SF-1 is truly a master regulator for the production of steroidogenic cells from human BMCs.
\end{abstract}

Journal of Molecular Endocrinology (2007) 39, 343-350

\section{Introduction}

Steroidogenic factor 1 (SF-1) was initially identified as a ubiquitous transcription factor for steroidogenic genes (Yanase et al. 1991, Honda et al. 1993, Omura \& Morohashi 1995, Parker \& Schimmer 1997). SF-1 binds as a monomer to its responsive element located in the promoter regions of steroidogenic genes and enhances their transcriptional levels (Honda et al. 1993, Omura \& Morohashi 1995, Parker \& Schimmer 1997). In addition, the cAMP-protein kinase A signal pathway can strongly potentiate SF-1 transactivation activity (Fan et al. 2004). Since knockout mice for SF-1 show agenesis of both the adrenal glands and the gonads, as well as decreased expression levels of luteinizing hormone $(\mathrm{LH})$ and follicle-stimulating hormone (FSH) in the pituitary gonadotroph (Ingraham et al. 1994, Luo et al. 1994, Morohashi \& Omura 1996), SF-1 has been considered to be an essential factor for differentiation of the pituitaryadrenal or pituitary-gonadal axis. Dosage-sensitive sex reversal, adrenal hypoplasia congenital, critical region on the $\mathrm{X}$ chromosome gene 1 (DAX-1) is also an important factor for the development of this axis since patients with DAX-1 mutations show X-linked adrenal hypoplasia congenital (AHC) and hypogonadotropic hypogonadism (Muscatelli et al. 1994, Yanase et al. 1996). Thus, DAX-1 is considered to be involved in the differentiation process of the fetal adrenal gland. A tumor suppressor gene, Wilms' tumor 1 (WT1), was also proven to be related to urogenital development, including the development of the adrenal gland, from the findings of phenotypes in patients with mutations (Little \& Wells 1997) and knockout mice (Wagner et al. 2003). Although their exact roles in humans are unknown, many other factors, such as pre-B-cell leukemia homeobox 1 (PBX-1; Schnabel et al. 2003), CBP/p300-interacting transactivator with Glu/Asp-rich C-terminal domain 2 (CITED2; Bamforth et al. 2001), wingless type MMTV integration site family member 4 (WNT4; Vainio et al. 1999), M33 (Katoh-Fukui et al. 1998), fibroblast growth factor 9 (Colvin et al. 2001), and GATA binding protein 4 (Tevosian et al. 2002), are also known to be involved in 
adrenal or gonadal development since disruption or overexpression of these genes in mice causes sex reversal or delayed sexual differentiation (Katoh-Fukui et al. 1998, Vainio et al. 1999, Bamforth et al. 2001, Colvin et al. 2001, Schnabel et al. 2003, Hammer et al. 2005).

In animal experiments, adrenocortical tissue has been successfully regenerated through xenotransplantation of cloned adrenocortical cells (Thomas et al. 1997), suggesting that the intraadrenal stem cells required for such tissue formation may be present in the adrenal cortex. Stable expression of SF-1 has been shown to direct embryonic stem cells toward the steroidogenic lineage. However, this steroidogenic capacity was very limited since progesterone was the only steroid produced in the presence of an exogenous substrate, 20 $\alpha$-hydroxycholesterol (Crawford et al. 1997).

Bone marrow cells (BMCs) may contribute to the regeneration of hematopoietic or mesenchymal lineages in multiple organs (Pittenger et al. 1999, Song \& Tuan 2004). Therefore, we tested whether the introduction of SF-1 into mouse BMCs could produce steroidogenic cells (Gondo et al. 2004). We expanded a relatively pure BMC population by culturing the cells for 120-180 days, and then demonstrated that adenovirus-mediated forced expression of SF-1 in long-term cultured BMCs can produce steroidogenic cells with the capacity for de novo synthesis of various steroid hormones in response to adrenocorticotropic hormone (ACTH). Similar to our data, a recent report demonstrated that cAMP stimulation of a cloned mouse BMC line, KUM9, and human BMC line, hMSC-hTERT-E6/E7, stably expressing SF-1 induced cell differentiation into cells with a testis- and adrenal-type steroidogenic capacity respectively (Yazawa et al. 2006). These results suggest a promising utility of BMCs as a regenerative source of steroidogenic cells.

For the future clinical application of SF-1 in autologous cell transplantation therapy for patients with steroid hormone deficiency, it is very important to examine its effect on human BMCs. In this regard, we applied this technique to human BMCs and compared their steroidogenic profiles with those of mouse BMCs. In addition, we investigated the impacts of several known factors, WT1, DAX-1, PBX-1, CITED2, and WNT4, which are involved in adrenal and/or gonadal development, to determine whether the single introduction of each factor may also be sufficient to transform human BMCs into steroidogenic cells.

\section{Materials and methods}

\section{Construction of the adenovirus and the lentivirus vectors}

A recombinant adenovirus vector derived from the human type 5 adenovirus using a commercially available Adenovirus Expression Vector Kit (TakaraBio Ltd, Shiga, Japan), containing bovine SF-1 cDNA (AdxbSF-1) or LacZ cDNA (Adx-LacZ) as a control, and was prepared as previously described (Gondo et al. 2004). Full-length human cDNAs for SF-1, WT1, DAX-1, PBX-1, CITED2, and WNT4 were cloned by reverse transcriptase-polymerase chain reaction (RT-PCR) using appropriate tissue total RNA with primers based on the GenBank database, subcloned into pCR-Blunt II-TOPO vector (Invitrogen) and then the entire sequences of these constructs were verified by sequencing. Each human cDNA was inserted into lentiviral vector (CS-CDF-CG-PRE, RIKEN BioResource Center, Tsukuba, Japan) and the preparation of each recombinant lentivirus was performed according to the manufacturer's protocol.

\section{Culture of human mesenchymal BMCs and treatment with adenovirus or lentivirus}

Human bone marrow mononuclear cells purchased from Cambrex (East Rutherford, NJ, USA) were cultured in nonhematopoietic $(\mathrm{NH})$ Expansion medium (Miltenyi Biotec, Bergisch Gladbach, Germany) at $37^{\circ} \mathrm{C}$ in a $5 \%$ $\mathrm{CO}_{2}$ incubator for 4 weeks. Only adherent cells were used in the experiments. To test the capability for osteoblastic differentiation, $4.5 \times 10^{4}$ cells were plated on $35 \mathrm{~mm}$ dish, cultured in NH OsteoDiff medium (Miltenyi Biotec) for 10 days and stained with alkaline phosphatase (Gondo et al. 2004). Adipogenic differentiation was confirmed at a cell density of $7.5 \times 10^{4}$ cells $/ 35 \mathrm{~mm}$ dish following treatment with NH AdipoDiff medium (Miltenyi Biotec) for 21 days and stained with Oil red $\mathrm{O}$ to test the capacity of cells to differentiate into mature adipocytes (Song \& Tuan 2004).

Human BMCs $\left(10^{4}\right.$ cells/well $)$ were cultured on a collagen type I-coated 24-well plate, incubated in AdxbSF-1 or Adx-LacZ medium at $37^{\circ} \mathrm{C}$ for $1 \mathrm{~h}$, rinsed with PBS, and cultured for 7 days. Culture medium was then changed and BMCs were cultured for another 4 days for measurements of steroid contents. In addition, human BMCs $\left(10^{4}\right.$ cells/well) were cultured on a 24 -well plate, incubated in $\mathrm{NH}$ Expansion medium containing lentivirus vector at $37^{\circ} \mathrm{C}$ overnight, rinsed with PBS, and then cultured for another 10 days before being subjected to experiments.

\section{Measurements of the steroid content in the medium secreted from human BMCs}

Basal secretion levels of progesterone (P4), corticosterone (B), cortisol (F), aldosterone, testosterone $(\mathrm{T})$, and estradiol $\left(\mathrm{E}_{2}\right)$ secreted into the culture medium were measured using an EIA kit (Cayman Chemical, Ann 
Arbor, MI, USA). Dehydroepiandrosterone (DHEA) was measured using another EIA kit (Assay Designs, Ann Arbor, MI, USA). The detection limits of P4, B, F, aldosterone, $\mathrm{T}, \mathrm{E}_{2}$, and DHEA were $10,38,17,21,6,8$, and $15 \mathrm{pg} / \mathrm{ml}$ respectively. The secretions of $\mathrm{F}$ and $\mathrm{T}$ into the medium were also confirmed in the presence or absence of synthetic 1-24 ACTH (Shionogi Co., Osaka, Japan) or human chorionic gonadotropin (hCG; Aska Co. Ltd, Tokyo, Japan). Human BMCs were infected with Adx-bSF-1 (multiplicity of infection, MOI $=20$ ) and cultured for 7 days. Culture medium was then collected at day 11 , and the cells were stimulated with $2.4 \mu \mathrm{MACTH}$ or $10 \mathrm{mU} / \mathrm{ml} \mathrm{hCG}$. Every 3-4 days, culture medium was collected for measurement of $\mathrm{F}$ and $\mathrm{T}$ concentrations and cells weretreated with ACTH or hCG.

\section{Quantitative real-time PCR}

We performed quantitative analysis of the mRNA expression levels of ACTH receptor (ACTH-R), LH receptor (LH-R), and various steroidogenic enzymes including P450scc, 3ß-hydroxysteroid dehydrogenase (3ß-HSD), P450c21, P450c11, P450c17, P450ald, $17 \beta$-HSD type 3 , and P450arom, by real-time PCR using a LightCycler (Roche Diagnostics $\mathrm{GmbH}$ ) as described previously (Gondo et al. 2004). We isolated total RNA from cultured human BMCs using an RNeasy Mini kit (Qiagen, GmbH). We synthesized first-strand complimentary DNA using $1 \mu \mathrm{g}$ total RNA as a template with QuantiTect RT kit (Qiagen) and carried out PCR in a LightCycler according to the manufacturer's instructions. We used the carefully designed sense/ antisense primers of P450scc, 3 $\beta$-HSD, P450c21, P450c11, P450ald, 17 $\beta$-HSD type 3, ACTH-R, LH-R, $\mathrm{P} 450$ arom, and $\beta$-actin (TakaraBio). PCR primers and conditions are available on request. Threshold values were obtained where fluorescent intensity was in the geometric phase of amplification, as determined with LightCycler Software Ver. 3.5. Products were verified on $2 \%$ agarose gels. We verified the nucleotide sequences of each PCR product by direct sequencing using the appropriate primers. Relative expression levels of the mRNAs were calibrated to those of $\beta$-actin.

\section{Flowcytometry}

The protocol essentially followed a previously described method (Hirase et al. 2000). Briefly, $1 \times 10^{5}$ BMCs were incubated with either phycoerythin (PE)-conjugated anti-human c-kit, CD11b, CD31, CD34, CD44, CD45, and CD105 monoclonal antibodies (BD Biosciences, Franklin Lakes, NJ, USA) or an isotype-matched PE-conjugated mouse IgG (BD Biosciences) for $30 \mathrm{~min}$ at $4^{\circ} \mathrm{C}$. The cells were finally analyzed on a FACScan flowcytometer (BD Biosciences).

\section{Immunofluorescence cell staining}

We conducted immunofluorescence cell staining of BMCs using goat anti-ACTH-R and anti-LH-R antibodies (Santa Cruz Biotechnology Inc., Santa Cruz, CA, USA), rabbit antibody against bovine SF-1 (kindly provided by Prof. Morohashi, Kyushu University, Japan), Alexa Fluor 488 donkey anti-rabbit IgG and Alexa Fluor 594 rabbit anti-goat IgG (Molecular Probes, Eugene, OR, USA). Inoculated cells were plated onto CC2-treated chamber slides (Nalge Nunc International Co., Naperville, IL, USA), cultured for 3 days and fixed with $4 \%$ paraformaldehyde at $4{ }^{\circ} \mathrm{C}$ for $1 \mathrm{~h}$. Immunofluorescence cell staining was then performed according to the manufacturer's protocol. The fluorescence was observed using fluorescence microscopy Biozero (Keyence, Tokyo, Japan).

\section{Immunoblotting}

Human BMCs were washed with PBS and lysed in CelLytic M cell lysis reagent (Sigma). A total of $20 \mu \mathrm{g}$ protein was subjected to SDS-PAGE and transferred onto a PVDF membrane (Bio-Rad Laboratories). Western blotting was performed according to the manufacturer's protocol. Protein levels were visualized using an ECL Plus kit (GE Healthcare, Buckinghamshire, UK) and a LAS3000 detector (Fuji Film, Tokyo, Japan).

\section{Statistical analysis}

One-factor ANOVA was used for statistical evaluation. $P<0.05$ was considered to be statistically significant.

\section{Results}

Flowcytometric analyses revealed that the human BMCs prepared for steroidogenic transformation were negative for the surface markers including CD45 (leukocyte marker), CD11b (monocyte/macrophage marker), CD34, c-kit (hematopoietic stem cell markers), and CD31 (endothelial cell marker) but positive for CD44 and CD105 (potential marker for mouse mesenchymal cells; data not shown). Although the BMCs in our experiment still constitute a heterogeneous population, the analysis of cell surface markers strongly suggested the possibility that the steroidproducing cells originate from multipotent and immature stem cells. Importantly, human BMCs differentiated into an osteoblastic phenotype and adipocyte phenotype following respective proper treatment (data not shown), suggesting that the character of the human BMCs may be much closer to that of mesenchymal BMC lineages. These results suggest that 
steroid-producing cells originate from multipotent and immature stem cells.

The above human mesenchymal BMCs were inoculated with Adx-bSF-1 (MOI=0, 10, 50, 100, and 200) and cultured for 3,7 , and 11 days. We analyzed the expression level of SF-1 using antibody against SF-1. The bovine SF-1 expression was observed predominantly in the nucleus of most of the cultured human BMCs even by minimal infection at MOI of 10 (Fig. 1A). The expression level seems to be increased in a MOIdependent manner as shown by immunofluorescence staining at day 3 (Fig. 1A) and by western blot analysis at day 11 (Fig. 1B). Thus, the following experiments were performed 10 days after infection. As in mouse cells (Gondo et al. 2004), throughout these experiments, induction of endogenous SF-1, namely the expression of human SF-1 was not observed by real-time PCR (data not shown).

First, to quantify the basal secretion levels of steroid hormones, cultured medium (day 11) of human BMCs infected with Adx-bSF-1 or Adx-LacZ was analyzed. Cells

A

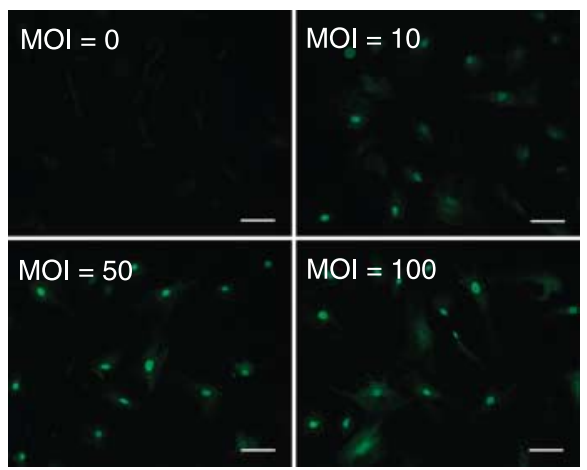

B

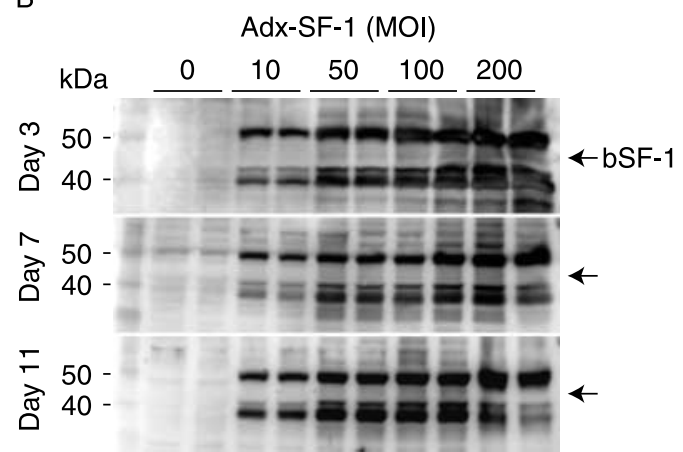

Figure 1 SF-1 expression in human BMCs infected with adenovirus vector. Human BMCs were inoculated with Adx-bSF-1 (MOI =0, 10, 50, 100, or 200) and cultured for 3, 7, and 11 days. To analyze the expression level of bSF-1, infected cells were subjected to $(A)$ immunofluorescence staining and total cell lysate was subjected to (B) immunoblot analysis using an antibody against SF-1 (please see Materials and methods). The bar indicates a $100 \mu \mathrm{m}$ scale. were infected with Adx-bSF-1 and cultured for 7 days. The steroid content accumulating in the medium over the next 4 days was then measured. Progesterone (P4) secretion was increased with a dependency on MOI of Adx-bSF-1, namely SF-1 expression level (data not shown). The secretions of P4, corticosterone (B), cortisol (F), aldosterone, DHEA, testosterone (T), and $\mathrm{E}_{2}$ into the medium from human BMCs infected with Adx-bSF-1 $(\mathrm{MOI}=50)$ were increased when compared with non-infected cells $(\mathrm{MOI}=0)$ or cells infected with Adx-LacZ (MOI=50; Fig. 2A). To investigate whether steroid synthetic enzymes were upregulated in human BMCs infected with Adx-bSF-1, the mRNA levels of such enzymes were analyzed by real-time PCR (Fig. 2B). The expression levels of P450scc, 3 $\beta$-HSD, P450c21, P450c11, P450c17, 17 $\beta$-HSD type 3, and P450arom mRNAs relative to $\beta$-actin, expressed as the ratio to those of non-infected BMCs $(\mathrm{MOI}=0)$, were significantly increased with increasing SF-1 expression level; cells infected with Adx-LacZ did not show such a phenomenon (Fig. 2B). Despite the presence of aldosterone production, we could not detect P450ald by real-time PCR.

In Adx-bSF-1-infected human BMCs, the mRNA expression levels of both ACTH-R and LH-R relative to $\beta$-actin were increased in a MOI-dependent manner (Fig. 3A), while those in cells infected with Adx-LacZ was undetectable. Next, human BMCs were infected with Adx-bSF-1 or Adx-LacZ $(\mathrm{MOI}=200)$, and 3 days later they were stained with specific antibodies against the ACTH-R, LH-R, or SF-1. As a result, both receptors were detected in SF-1-positive cells, suggesting actual inductions of both receptors by SF-1 (Fig. 3B). At 3 day intervals after infection of the cells with Adx-bSF-1 $(\mathrm{MOI}=20)$, the cells were stimulated with $2 \cdot 4 \mu \mathrm{M}$ ACTH or $10 \mathrm{mU} / \mathrm{ml}$ hCG. After stimulation the medium was collected for the measurement of steroid content. The secretion of cortisol into the medium was increased in response to both $2 \cdot 4 \mu \mathrm{M}$ ACTH and $10 \mathrm{mU} / \mathrm{ml}$ hCG stimulation (Fig. 3C). The maximum response of cortisol to ACTH or hCG was observed on day 21 , when cortisol production was increased by $9 \cdot 5-$ and 6-fold in response to ACTH and hCG respectively. Likewise, the secretion of testosterone was increased in response to both hCG and ACTH stimulation, and the maximum response was obtained at day 18 (Fig. 3C). Together, these data indicate a capability of human mesenchymal BMCs to be transformed into steroidogenic cells, which are capable of responding to ACTH and $\mathrm{LH}$.

Finally, we tested the effects of several factors that are reported to be associated with adrenal and/or gonadal development, mainly based on the results of human or mouse phenotypes due to a complete or partial lack of each factor (Muscatelli et al. 1994, Yanase et al. 1996, Vainio et al. 1999, Bamforth et al. 2001, Schnabel et al. 
A
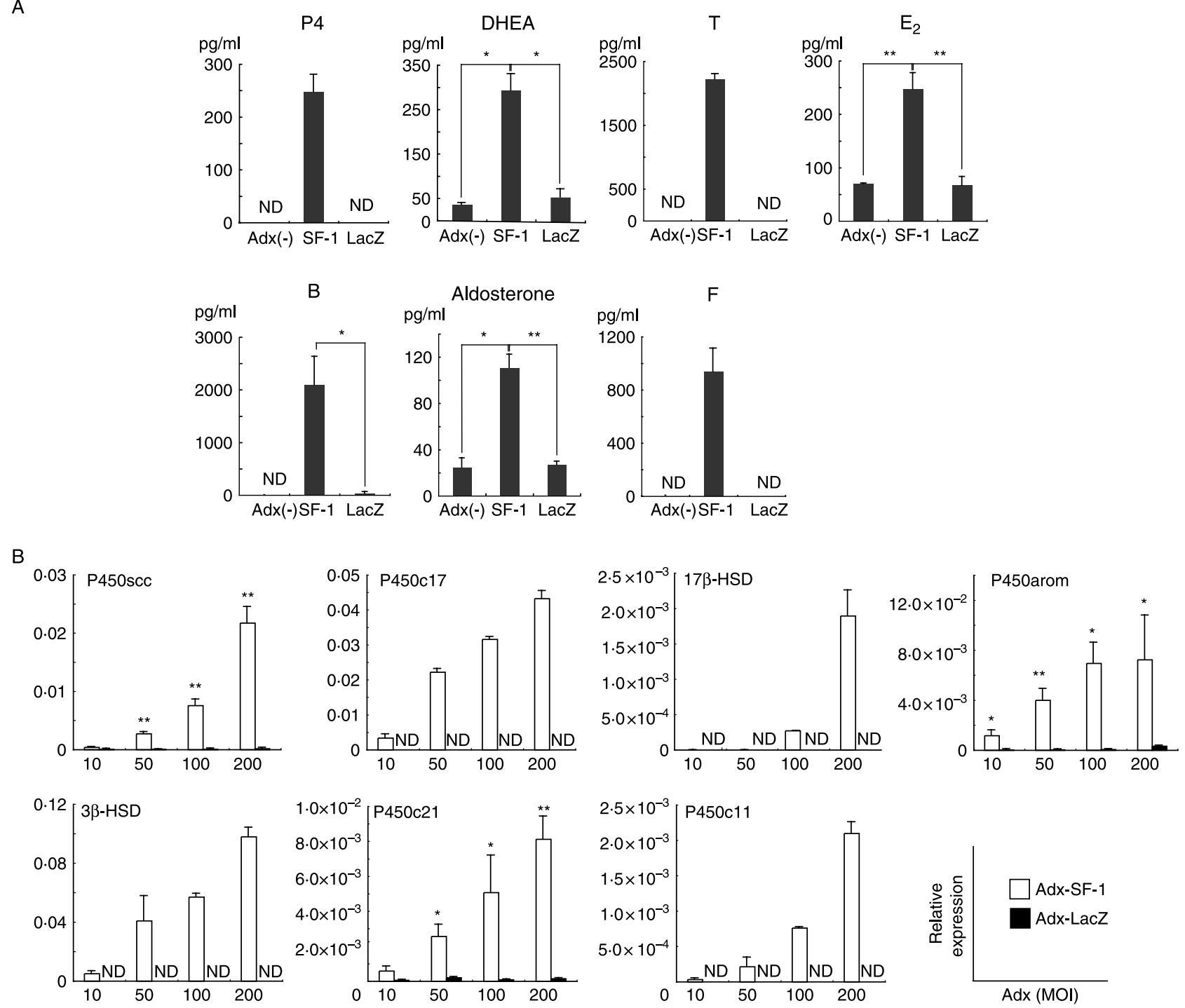

Figure 2 Basal steroid production and mRNA expression levels of steroidogenic enzymes in human BMCs infected with Adx-bSF-1. Cells were infected with Adx-bSF-1 and cultured for 7 days. The steroid content accumulated in the medium over the next 4 days was then measured. (A) Basal secretion of progesterone (P4), corticosterone (B), aldosterone, cortisol (F), DHEA, testosterone (T), and estradiol $\left(E_{2}\right)$ into the medium from the human BMCs infected with Adx-bSF-1 $(\mathrm{MOI}=0,50)$ or Adx-LacZ $(\mathrm{MOI}=50)$. $(B)$ Real-time

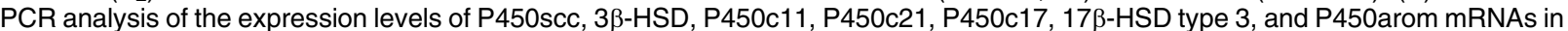
human BMC cells infected with Adx-bSF-1 or Adx-LacZ. The mRNA expression levels relative to the levels of $\beta$-actin are expressed. Values represent the means \pm s.D. $(n=3) .{ }^{\star} P<0 \cdot 05,{ }^{\star \star} P<0 \cdot 01$

2003). The tested factors were SF-1 as a positive control, GFP as a negative control, WT1, DAX-1, PBX-1, CITED2, and WNT4. WT1 encodes a zinc finger transcription factor. Alternative splicing of exon 5 results in a 17 amino acid insertion, and alternative splicing of exon 9 produces an insertion three amino acid Lys-Thr-Ser (KTS). Alternative splicing these two sites gives rise to four different protein isoforms designated $\mathrm{A}, \mathrm{B}, \mathrm{C}$, and $\mathrm{D}$; or $(-/-),(+/-)$, $(-/+)$, and $(+/+)$, representing the presence or absence of exon 5 and KTS respectively (Haber et al.
1991). In this experiment, for more convenience in the preparation or infection of cells with vector, cells were infected with lentivirus containing each factor. In a preliminary experiment, the expression of each factor was confirmed by real-time PCR or western blotting of the respective infected cells, 10 days after infection (data not shown). Therefore, 10 days after infection, the medium was changed and the $\mathrm{F}$ and $\mathrm{T}$ content in the medium on each of the next 4 days (day 11-14 days) were measured; the expression levels of P450scc in cells at day 14 were also determined by real-time PCR. Introduction 
A

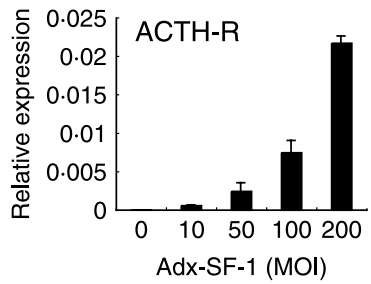

B
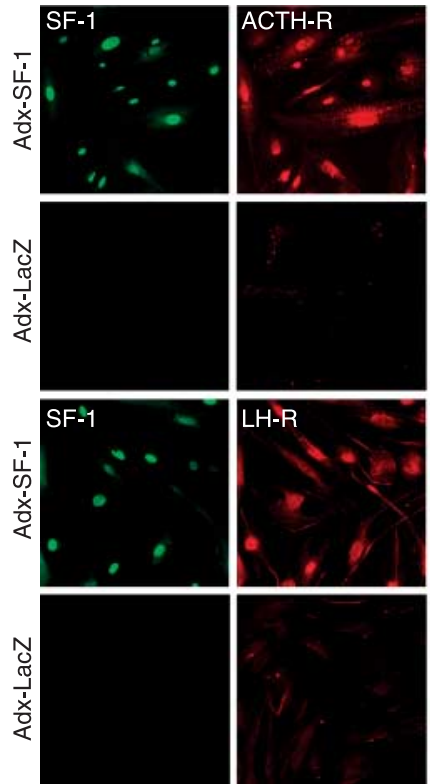
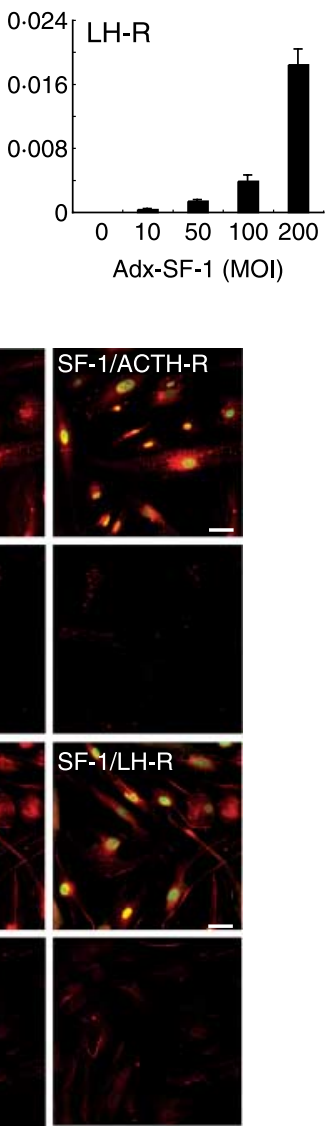

C
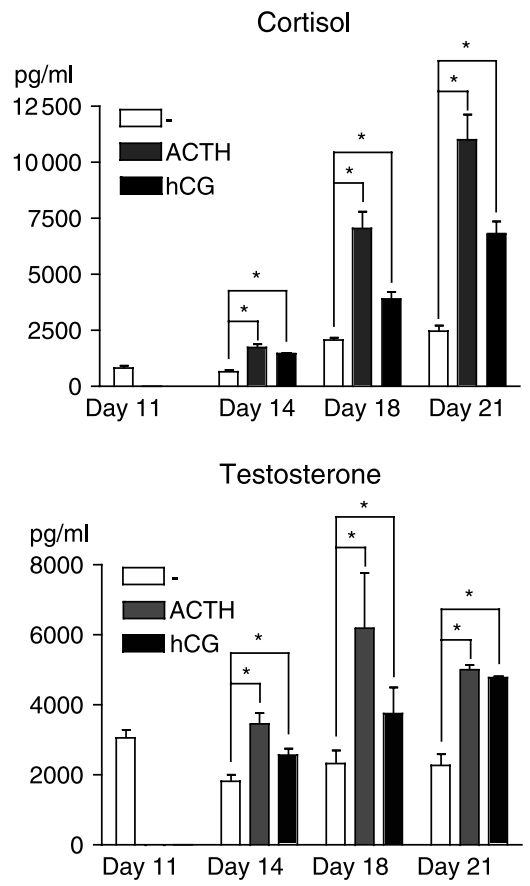

Figure 3 Expression of ACTH-R and LH-R and responsiveness to ATCH and LH in cultured human BMCs. (A) Human BMCs infected with Adx-bSF-1 or Adx-LacZ were cultured for 11 days and then the expression levels of ACTH-R and LH-R were determined by real-time PCR. (B) Human BMCs infected with Adx-bSF-1 or $\mathrm{Adx}-\mathrm{LacZ}(\mathrm{MOI}=200)$ were cultured for 3 days, and then the expression levels of $\mathrm{ACTH}-\mathrm{R}$ and $\mathrm{LH}-\mathrm{R}$ were determined by immunofluorescence staining using antibodies against SF-1 and ACTH-R or LH-R The bar indicates a $50 \mu \mathrm{m}$ scale. (C) The effect of ACTH and LH on the secretion of cortisol and testosterone respectively, from cultured human BMCs. Human BMCs were infected with Adx-bSF-1 $(\mathrm{MOI}=20)$ and cultured for 7 days. Culture medium was then collected at day 11 for measurement of basal secretions of steroids, and the cells were stimulated with $2.4 \mu \mathrm{M}$ ACTH or $10 \mathrm{mU} / \mathrm{ml} \mathrm{hCG}$. Every 3-4 days, culture medium was collected for measurement of cortisol and testosterone concentrations and cells were treated with ACTH or hCG. The secretion of cortisol or testosterone into the medium was increased in response to ACTH and hCG stimulation respectively. Values represent the means \pm s.D. $(n=3) . P<0.05$ against the control (absence of ACTH or hCG).

of SF-1 only into human BMCs induced a $4 \cdot 7$-fold induction of P450scc expression when compared with GFP, and caused 11.3- and 12.5-fold increase in the secretion of $\mathrm{F}$ and $\mathrm{T}$ into the medium respectively. Single introductions of WT1 $(-/-,-/+,+/-$, and $+/+)$, DAX-1, PBX-1, CITED2, and WNT4 did not produce any such effects (data not shown).

\section{Discussion}

Although the character of human mesenchymal stem cells has not been fully clarified and remains controversial, steroid-producing cells may originate from multipotent and immature stem cells. Importantly, human cultured BMCs were able to differentiate into an osteoblastic or adipocyte phenotype following appropriate treatments, suggesting that the character of the prepared human BMCs may be much closer to mesenchymal BMC lineages. Under such conditions, we demonstrated, for the first time, that human BMCs infected with Adx-bSF-1 can produce significant amounts of progesterone, corticosterone, cortisol, DHEA, testosterone, and estradiol, owing to the expression of mRNAs for P450scc, 3 $\beta$-HSD, P450c11, P450c21 P450c17, 17 $\beta$-HSD type 3, and P450arom, as detected by RT-PCR. Most of the profiles of steroidogenic enzymes were quite similar to those in mouse 
BMCs (Gondo et al. 2004), except for the finding that P450c21 is expressed in human cells but not in mouse cells. Although we observed a significant amount of aldosterone production, we could not show the expression of P450ald by real-time PCR (data not shown). Thus, we cannot completely conclude a capability of human BMCs for aldosterone production. Further studies are needed on this point including the optimization of PCR conditions. As in mouse cells (Gondo et al. 2004), the steroid profile of cultured human BMCs showed a mixed pattern of adrenal and gonadal steroidogenesis, namely the simultaneous production of cortisol and sex steroids. This finding matches well an established concept that common precursor cells divide into two distinct characteristic cell types, designated adrenal precursor cells and bipotential gonadal precursor cells, and finally develop into the adrenal cortex or testes and ovaries, respectively, under the regulation of many transcriptional factors, including SF-1 (Yanase et al. 2006).

A feature of human steroidogenic cells that makes them remarkably distinct from mouse cells is the clear induction of ACTH-R and LH-R following the introduction of SF-1. Mouse BMCs originally expressed ACTH-R without introduction of SF-1, namely predominant expression of an adipose tissue type of ACTH-R (Kubo et al. 2004), rather than an adrenal tissue type of ACTH-R (Cammas et al. 1997). The introduction of SF-1 itself did not increase ACTH-R expression in mouse BMCs, since the promoter region of the adipose tissue type ACTH-R gene does not contain SF-1 sites, unlike the promoter of the adrenal type ACTH-R gene (Cammas et al. 1997). On the other hand, the gene encoding the human ACTH receptor does not seem to produce extra-adrenal type isoform from its gene structure (Kubo et al. 2004) and produces only adrenal type receptor, which can be responsive to SF-1; mRNA for this receptor was expressed in human BMCs (data not shown). Similarly, LH-R was dramatically induced in human BMCs, but not in mouse BMCs. The human LH-R gene promoter also contains a potential SF-1 site, although binding SF-1 to this site is not proven (Geng et al. 1999). There may be an unknown SF-1 induced factor that promotes the expression of LH-R. Anyway, the findings of good induction of ACTH-R and LH-R in human BMCs and responsiveness of these cells to ACTH and LH are physiologically important when we consider a future clinical application for autologous BMC transplantation into patients with steroid insufficiency.

In humans, sex-determining region Y (SRY) initiates the testis-determining pathway by inducing the expression of a series of downstream factors, including SF-1, WT1, DAX-1, SRY-related genes HMG-box 9, and several other factors, thus resulting in a male phenotype (Yanase et al. 2006). In this pathway, SF-1 has been considered to be a master regulator that controls the overall stream of the developmental mechanism. WT1 seems to be of interest because, in contrast to the adrenal agenesis seen in mice lacking all Wt1 transcript (Wagner et al. 2003), analyses of mice with isoformspecific disruption of Wt1 have shown different roles for two alternatively spliced transcripts the - KTS and + KTS isoforms (Hammes et al. 2001). Namely, selective inactivation of the + KTS isoform did not impair ovary development, but rather impaired testis and male sex differentiations. PBX-1 is a homeodomain protein that contributes to adrenal development since, PBX-1 KO mice, which die in utero due to defects in multiple organs, completely lacked adrenal glands and had impaired testis development associated with decreased proliferation in the urogenital ridges (Schnabel et al. 2003). A coactivator CITED2 coregulates genes that are essential for adrenal development since its KO mice were found to have adrenal agenesis (Bamforth et al. 2001). Wnt proteins act via the frizzled receptor family to initiate a canonical cascade of intracellular signals leading to $\beta$-catenin accumulation in the nucleus and subsequent transactivation of downstream target genes (Tolwinski \& Wieschaus 2004). Disruption of WNT4 in mice causes a marked masculinization of XX females with absence of the female Mullerian duct and persistence of the male Wolffian duct derivatives due to excess gonadal testosterone synthesis, and abnormal differentiation of the definitive zone in the adrenal gland (Vainio et al. 1999).

While, admittedly, the present experiment was preliminary in that we have not dealt with all known factors, we tested whether the above factors, which are closely associated with sexual differentiation including adrenal and/or gonadal development, could transform human BMCs into steroidogenic cells. Our finding that only SF-1 among SF-1, WT1, DAX-1, PBX-1, CITED2, and WNT4 could transform human BMCs into steroidogenic cells suggested that SF-1 is truly a master regulator, even for the production of steroidogenic cells from human mesenchymal BMCs. However, it is also important to test the functional relationship between SF-1 and other factors involved in steroidogenesis by multiple transfections, because sexual differentiation takes place in a series of cascades of various factors (Yanase et al. 2006). Such detailed experiments are currently underway in our laboratory.

In summary, we showed that SF-1 could transform human bone marrow mesenchymal cells into steroidogenic cells. This finding is expected to develop extensively in the near future, possibly leading to the development of therapies to relieve the many patients who are forced to take hormone replacement therapy. 


\section{Acknowledgements}

We acknowledge the support of Health and Labour Sciences Research Grant and grant of Ministry of Education, Culture, Sports, Science and Culture (No. 16086207: Molecular mechanisms of sex differentiation). The authors declare that there is no conflict of interest that would prejudice the impartiality of this scientific work.

\section{References}

Bamforth SD, Braganca J, Eloranta JJ, Murdoch JN, Marques FI, Kranc KR, Farza H, Henderson DJ, Hurst HC \& Bhattacharya S 2001 Cardiac malformations, adrenal agenesis, neural crest defects and exencephaly in mice lacking Cited2, a new Tfap2 co-activator. Nature Genetics 29 469-474.

Cammas FM, Pullinger GD, Barker S \& Clark AJ 1997 The mouse adrenocorticotropin receptor gene: cloning and characterization of its promoter and evidence for a role for the orphan nuclear receptor steroidogenic factor 1. Molecular Endocrinology 11 867-876.

Colvin JS, Green RP, Schmahl J, Capel B \& Ornitz DM 2001 Maleto-female sex reversal in mice lacking fibroblast growth factor 9. Cell 104 875-889.

Crawford PA, Sadovsky Y \& Milbrandt J 1997 Nuclear receptor steroidogenic factor 1 directs embryonic stem cells toward the steroidogenic lineage. Molecular and Cellular Biology 17 3997-4006.

Fan W, Yanase T, Wu Y, Kawate H, Saitoh M, Oba K, Nomura M, Okabe T, Goto K, Yanagisawa J et al. 2004 Protein kinase A potentiates adrenal 4 binding protein/steroidogenic factor 1 transactivation by reintegrating the subcellular dynamic interactions of the nuclear receptor with its cofactors, general control nonderepressed-5/transformation/ transcription domain-associated protein, and suppressor, dosagesensitive sex reversal-1: a laser confocal imaging study in living KGN cells. Molecular Endocrinology 18 127-141.

Geng Y, Tsai-Morris CH, Zhang Y \& Dufau ML 1999 The human luteinizing hormone receptor gene promoter: activation by Spl and Sp3 and inhibitory regulation. Biochemical and Biophysical Research Communications 263 366-371.

Gondo S, Yanase T, Okabe T, Tanaka T, Morinaga H, Nomura M, Goto K \& Nawata H 2004 SF-1/Ad4BP transforms primary long-term cultured bone marrow cells into ACTH-responsive steroidogenic cells. Genes to Cells 9 1239-1247.

Haber DA, Sohn RL, Buckler AJ, Pelletier J, Call KM \& Housman DE 1991 Alternative splicing and genomic structure of the Wilms tumor gene WT1. PNAS 88 9618-9622.

Hammer GD, Parker KL \& Schimmer BP 2005 Minireview: transcriptional regulation of adrenocortical development. Endocrinology 146 1018-1024.

Hammes A, Guo JK, Lutsch G, Leheste JR, Landrock D, Ziegler U, Gubler MC \& Schedl A 2001 Two splice variants of the Wilms' tumor 1 gene have distinct functions during sex determination and nephron formation. Cell 106 319-329.

Hirase N, Yanase T, Mu Y, Muta K, Umemura T, Takayanagi R \& Nawata H 2000 Thiazolidinedione suppresses the expression of erythroid phenotype in erythroleukemia cell line K562. Leukemia Research 24 393-400.

Honda S, Morohashi K, Nomura M, Takeya H, Kitajima M \& Omura T 1993 Ad4BP regulating steroidogenic P-450 gene is a member of steroid hormone receptor superfamily. Journal of Biological Chemistry 268 7494-7502.

Ingraham HA, Lala DS, Ikeda Y, Luo X, Shen WH, Nachtigal MW, Abbud R, Nilson JH \& Parker KL 1994 The nuclear receptor steroidogenic factor 1 acts at multiple levels of the reproductive axis. Genes and Development 8 2302-2312.
Katoh-Fukui Y, Tsuchiya R, Shiroishi T, Nakahara Y, Hashimoto N, Noguchi K \& Higashinakagawa T 1998 Male-to-female sex reversal in M33 mutant mice. Nature 393 688-692.

Kubo M, Shimizu C, Kijima H, Nagai S \& Koike T 2004 Alternate promoter and $5^{\prime}$-untranslated exon usage of the mouse adrenocorticotropin receptor gene in adipose tissue. Endocrine Journal 51 25-30.

Little M \& Wells C 1997 A clinical overview of WT1 gene mutations. Human Mutation 9 209-225.

Luo X, Ikeda Y \& Parker KL 1994 A cell-specific nuclear receptor is essential for adrenal and gonadal development and sexual differentiation. Cell 77 481-490.

Morohashi KI \& Omura T 1996 Ad4BP/SF-1, a transcription factor essential for the transcription of steroidogenic cytochrome P450 genes and for the establishment of the reproductive function. FASEB Journal 10 1569-1577.

Muscatelli F, Strom TM, Walker AP, Zanaria E, Recan D, Meindl A, Bardoni B, Guioli S, Zehetner G, Rabl W et al. 1994 Mutations in the DAX-1 gene give rise to both X-linked adrenal hypoplasia congenita and hypogonadotropic hypogonadism. Nature 372 672-676.

Omura T \& Morohashi K 1995 Gene regulation of steroidogenesis. Journal of Steroid Biochemistry and Molecular Biology 53 19-25.

Parker KL \& Schimmer BP 1997 Steroidogenic factor 1: a key determinant of endocrine development and function. Endocrine Reviews 18 361-377.

Pittenger MF, Mackay AM, Beck SC, Jaiswal RK, Douglas R, Mosca JD, Moorman MA, Simonetti DW, Craig S \& Marshak DR 1999 Multilineage potential of adult human mesenchymal stem cells. Science 284 143-147.

Schnabel CA, Selleri L \& Cleary ML 2003 Pbx1 is essential for adrenal development and urogenital differentiation. Genesis 37 123-130.

Song L \& Tuan RS 2004 Transdifferentiation potential of human mesenchymal stem cells derived from bone marrow. FASEB Journal 18 980-982.

Tevosian SG, Albrecht KH, Crispino JD, Fujiwara Y, Eicher EM \& Orkin SH 2002 Gonadal differentiation, sex determination and normal Sry expression in mice require direct interaction between transcription partners GATA4 and FOG2. Development 129 4627-4634.

Thomas M, Northrup SR \& Hornsby PJ 1997 Adrenocortical tissue formed by transplantation of normal clones of bovine adrenocortical cells in scid mice replaces the essential functions of the animals' adrenal glands. Nature Medicine 3 978-983.

Tolwinski NS \& Wieschaus E 2004 Rethinking WNT signaling. Trends in Genetics 20 177-181.

Vainio S, Heikkila M, Kispert A, Chin N \& McMahon AP 1999 Female development in mammals is regulated by Wnt-4 signalling. Nature 397 405-409.

Wagner KD, Wagner N \& Schedl A 2003 The complex life of WT1. Journal of Cell Science 116 1653-1658.

Yanase T, Simpson ER \& Waterman MR 199117 alpha-hydroxylase/ 17,20-lyase deficiency: from clinical investigation to molecular definition. Endocrine Reviews 12 91-108.

Yanase T, Takayanagi R, Oba K, Nishi Y, Ohe K \& Nawata H 1996 New mutations of DAX-1 genes in two Japanese patients with X-linked congenital adrenal hypoplasia and hypogonadotropic hypogonadism. Journal of Clinical Endocrinology and Metabolism 81 530-535.

Yanase T, Gondo S, Okabe T, Tanaka T, Shirohzu H, Fan W, Oba K, Morinaga H, Nomura M, Ohe K et al. 2006 Differentiation and regeneration of adrenal tissues: an initial step toward regeneration therapy for steroid insufficiency. Endocrine Journal 53 449-459.

Yazawa T, Mizutani T, Yamada K, Kawata H, Sekiguchi T, Yoshino M, Kajitani T, Shou Z, Umezawa A \& Miyamoto K 2006 Differentiation of adult stem cells derived from bone marrow stroma into Leydig or adrenocortical cells. Endocrinology 147 4104-4111.

Received in final form 10 September 2007

Accepted 15 September 2007

Made available online as an Accepted Preprint

20 September 2007 